GUANG YUAN, Ph.D. candidate ${ }^{1,3,4}$

E-mail: yguang@emails.bjut.edu.cn

DEWEN KONG, Ph.D. 1,3,4

E-mail: kongdw@bjut.edu.cn

LISHAN SUN, Ph.D. . $^{1,3,4}$

(Corresponding author)

E-mail:1ssun@bjut.edu.cn

WEI LUO, Ph.D. ${ }^{2}$

E-mail: luowei@bucea.edu.cn

YAN XU, Ph.D. $1,3,4$

E-mail: yxu@bjut.edu.cn

${ }^{1}$ Beijing Key Laboratory of Traffic Engineering

Beijing University of Technology

100 Ping Le Yuan, Chaoyang District

Beijing, 100124, China

${ }^{2}$ Beijing University of Civil Engineering and Architecture

No.15 Yongyuan Road, Daxing District

Beijing, 00044, China

${ }^{3}$ Beijing Laboratory for Urban Mass Transit

100 Ping Le Yuan, Chanoyang District Beijing

100124, China

${ }^{4}$ Engineering Research Center of Digital Community

Ministry of Education, 100 Ping Le Yuan

Chanoyang District Beijing, 100124, China
Traffic Planning

Original Scientific Paper

Submitted: 4 Mar. 2020

Accepted: 6 July 2020

\title{
CONNECTIVITY CONTRIBUTION TO URBAN HUB NETWORK BASED ON SUPER NETWORK THEORY - CASE STUDY OF BEIJING
}

\begin{abstract}
With the rapid development of urbanization in China, the number of travel modes and urban passenger transportation hubs has been increasing, gradually forming multi-level and multi-attribute transport hub networks in the cities. At the same time, Super Network Theory (SNT) has advantages in displaying the multi-layer transport hubs. The aim of this paper is to provide a new perspective to study connectivity contribution of potential hubs. Urban transport hubs are ranked through topological features based on Hub Super Network (HSN). This paper proposes two indexes based on Super-Edge (SE), Zero Hub Degree of SE (ZHDoSE) and a number of shared SEes (NSSE), respectively. Then, a case study was conducted in Beijing, which considers four combinations to study the influence of transport modes and subway lines on connectivity. The results show that no-normalization strengthens the contribution of transport modes and subway lines on connectivity. Besides, the transport mode contributes a lot to the connectivity. However, elements normalization strengthens the subway lines under $\mathrm{ZH}$ DoSE reciprocal. In addition, various weights of $\mathrm{ZH}-$ DoSE and NSSE have different influences on the recognition results of SEes in HSN.
\end{abstract}

\author{
KEYWORDS \\ super network; super-edge; transportation hub; \\ connectivity.
}

\section{INTRODUCTION}

In recent years, the process of urbanization has had a leap-forward development in China, and numerous modes of transportation have emerged to meet the people's growing demands for travelling, which has improved the urban quality of life and urban operational efficiency [1]. At the same time, multimodal traveling makes people converge at multimodal transfer points, which brings pressure to the transfer hubs in a city. Moreover, frequent occurrence of incidents has directly affected people's travels and commutes, even the economic development. Recently, scholars [2-6] have carried out the analyses of topological features that have great impacts on the characteristics of a network. Therefore, good understanding of the topological features of a network could guide hubs management operations and take preventive measures effectively. 
Of course, like many topological networks in transportation [6-11], the features of urban hubs have been studied before by complex network in which the urban hubs are taken as single nodes. As urban hubs contain several transport modes, the single complex network has defects in performing multimodal urban hubs. However, the multimodal network hub is much more complicated than single transportation hubs on complex network. More specifically, the hub has multiple transport modes and multiple track lines in a node, which means multi-tier attributes in the nodes. Furthermore, the transfer hub includes various facilities of several transport modes and connections of different facilities, which increases the complexity and difficulty in performance analysis on complex networks. In addition, the complexity is also brought by the increasing number of hubs and spatial distribution in a city. Hubs network will lose more multi-dimensional information when the hubs are simplified by a single complex network. Therefore, the urban hub network has the characteristics of multi-dimension, multi-level, multi-attribute, and multiplicity. It will lose much information when being analysed using a single network. For example, nodes just show one transport mode and link, failing to represent the relationship between travel modes and layers. The characteristics of urban hubs that are formed by the overlaying of several transport modes cannot be fully reflected by a single network. The study on hubs with multiple transport modes is rarely mentioned in the previous research. Therefore, there is an urgent need for a multi-dimensional method or model to interpret the structure of urban hubs and study the characteristics. Then, it can provide guidelines for steps to improve operational efficiency, enhance service capacity, and then reduce the economic losses.

Hub transmission efficiency is an important index to evaluate hub services, and high connectivity between hubs is an important guarantee for hub efficiency. The connectivity has a great relationship with node degree in complex networks [12-14]. Many studies have done this before and achieved fruitful results. However, most of the research lacked effective modelling and analysis of urban hub networks, ignored the importance of transport modes and edge characteristics in multi-level hub networks. For instance, passengers plan to leave Beijing by trains. The passenger has fewer options in Beigongdaximen subway station (Line 14 contains Beijing south railway station) than the one in Jiulongshan subway station (Line 7 includes Beijing west railway station and Line 14 includes Beijing south railway station), which shows that a single node in a complex network will ignore two subway lines in Jiulongshan subway station. Therefore, new indices should be proposed to represent the features since node degree cannot describe the connectivity of a multi-level hub network. Transport modes and subway lines have great effects on the efficiency of multi-layer transport hubs. Therefore, a new index combined with the number of transport modes and the number of subway lines is presented in this paper.

Though features of the complex network have been a hot topic with the widespread application on transportation [15-18], it is difficult to describe the characteristics of multi-layer travel hub networks. Meanwhile, the concept of Super Network Theory (SNT) was firstly proposed by Sheffi in 1985 [19], and then, it was defined by professor Nagurney as the network of a network [20]. Up to the present, the SNT has the advantage of providing new perspectives and methods and has been widely applied in many fields [21-24]. Of course, some applications can be also found in the field of transportation $[25,26]$. However, it is rarely mentioned from the perspective of urban transportation hubs. The SNT can bring new elements with multi-dimensional attributes and describe nodes and links multi-dimensionally. Besides, new elements proposed in SNT carry a lot of information; for example, the connections between multi-layer nodes and multi-dimensional connection of a single node. Therefore, the SNT has great potential in exploration and application in the field of urban hubs.

Based on the existing research, this paper attempts to build a multi-layer model by SNT and provide appropriate indices to describe the connectivity properly. The SNT model can provide a new perspective for urban hub network and new elements. In this paper, multi-dimensional indicators are obtained and more information is represented by new elements. The paper then explores how transport modes and subway lines contribute to the connectivity of the urban hub network. According to SNT, the framework of urban Hub Super-Network (HSN) is built and new elements are presented. Besides, due to the uncertainty of combination, several combinations of transport modes and subway lines are discussed, and parameters are also discussed based on the HSN. The case study has been conducted in Beijing city. Hence, the main purpose of this paper is to explore the influence of the number 
of transport modes and the number of subway lines on connectivity. This knowledge could be utilized to provide useful guidance for urban transport hub operations and managements.

This paper is organized as follows. In the next Section, the HSN methods are described. In Section 3 , the case study is conducted, and the results are presented. In the Section of the discussion, factors affecting the connectivity are analysed. In the last Section, conclusions are drawn.

\section{METHODS}

\subsection{Assumptions}

Based on SNT [27-29], this paper proposes several assumptions before modelling, which are helpful to abstract urban hub and form the HSN. Besides, the urban hubs in Beijing city have been taken into account in this paper, which helps to explain well and to understand the new elements presented in the HSN methods.

Subway is the basic transport means in HSN. According to the research by Wang [30], which has taken the potential catchment area of transit station into account, the coverage area of the transit stations accounts for about $90 \%$ of the total area of the fifth ring road region in Beijing. Besides, according to the report of the Beijing transportation development 2019 [31], the share of the subway transport mode in the total travel volume in Beijing equals $49.5 \%$, and the annual transport volume of rail transit is 3.85 billion passengers per year. According to the spatial distribution of stations and the volume of transportation, the subway can be used as the basic transport means. The complexity of the bus transport network in Beijing makes it difficult to quantify the impacts of the bus stations. Meanwhile, the bus network, with large volume and wide radiation area, increases the complexity of HSN and reduces the profit of the model. Hence, the subway can be the basic transport mode in the multimodal hub network, and the bus network is not considered in the HSN model of this paper.

\subsection{Modelling}

According to the assumptions presented above, four sub-networks are constructed in HSN, including airport sub-network, railway sub-network, highway passenger sub-network, and subway sub-network. The sketch map of the HSN is shown in Figure 1 [21].
Sub-nodes that are labelled 0 are assigned to sub-network: due to the lack of certain mode(s), the hub nodes cannot reveal the intact vertical relations between sub-nodes in corresponding sub-networks. For instance, the Beijing west railway station does not have an airport travel method, which means the corresponding sub-nodes in the airport sub-network are missing. Therefore, nodes with label 0 are introduced to build the connection between sub-networks if the corresponding sub-node is missing. The sub-nodes labelled 0 are allocated to each sub-network layer. In general, label 0 represents the missing modes of transportation in HSN.

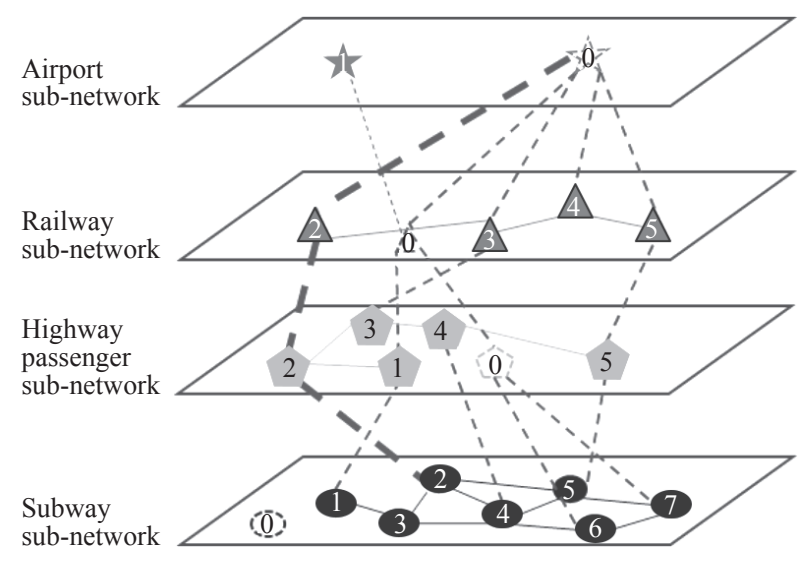

Figure 1 - Sketch map of HSN

Subway sub-network: the nodes in this sub-network are subway stations in the city, and the edges are the urban rail transit lines. The subway sub-network mainly services the travel demand within the city. An edge will be counted if two adjacent stations are linked by a line, and vice versa.

Highway passenger sub-network: in this sub-network, several critical highway passenger terminals (urban coach stations and municipal long-distance passenger transport hubs) are adopted, like Liuliqiao highway passenger terminal in Beijing. The edges in this sub-network indicate that there is a connection between sub-nodes by means of coaches.

Railway sub-network: the urban railway stations are the main sub-nodes in this sub-network, and the relationship between two nodes in the railway sub-network indicate the connection by railway lines within the city.

Airport sub-network: there is no question that the airport is the sub-node in this sub-network. In general, a city only has one airport in China. 
As Figure 1 shows, the relationships between inter-subnetwork and intra-subnetwork can be well clarified through the HSN. Therefore, there are two types of relationship lines; vertical lines and horizontal lines, respectively. The horizontal solid lines represent the connection of the sub-nodes of a certain sub-network, which shows the relationship between inner sub-networks. In addition, the colour of the line is the same as the colour of the sub-node in the same layer. The vertical lines are shown as dotted lines, which is the embodiment of the relationship between the sub-networks in the HSN. More specifically, the vertical lines indicate the connection of the same spatial location along different sub-networks.

\subsection{Indices of topological characteristics}

According to the application of SN [21, 23, 32], a new element of SN is the Super-Edge (SE). In the same way, SE equals the vertical line, such as the bold dotted vertical line (see Figure 1) in HSN. The SE connects a lot of sub-nodes in different layers, and the sub-nodes have two types based on whether they are labelled 0 or not. Therefore, SE has the potential to show the number of transport modes and the relationships between sub-networks that the complex network cannot show in nodes. In addition, another concept is represented in the HSN model, which is the sub-nodes of SE (SNSE) like the elements of the SE in other fields [21, 23, 32]. There is no doubt that the SNSE is a set of sub-nodes, which includes the sub-nodes in the SE and the sub-nodes connected to this SE in the subway sub-network. For instance, the line covered by the sub-nodes labelled 0 in airport sub-network, labelled 2 in railway sub-network, labelled 2 in highway passenger sub-network and labelled 2 in subway sub-network is a kind of SE which is named SE2. The sub-nodes in the line of SE2 plus the sub-nodes labelled 3, 4, and 5 in the subway sub-network are the SNSE of SE2.

As connectivity means the accessibility of nodes in a network [33-35], it has significant influence on the operational efficiency and level of service. In HSN, the connectivity is reflected by vertical and horizontal accessibility in the inter-layer and intra-layer. According to HSN, the SE and sub-nodes of SE show multi-dimensional relationship. Therefore, the indices will be proposed based on SE and sub-nodes of SE, which can well perform the effects of the number of transport modes and subway lines on connectivity.

The first index is named zero hub-node degrees of SEs (ZHDoSE), which calculates the amount of labelled 0 sub-nodes of SE. According to the definition, this index can well represent the number of transport modes in SE, which plays an essential role in the connectivity of hubs. This index is calculated by Equation 1:

$C_{0}^{e}=\left\{\begin{array}{lll}0 & \text { None } & \text { Labeled } 0 \\ 1 & 1 & \text { Labeled 0 } \\ 2 & 2 & \text { Labeled 0 } \\ 3 & 3 & \text { Labeled 0 }\end{array}\right.$

where $C_{0}^{e}$ represents the ZHDoSE of $e$-th SE. For example, SE2 has only one sub-node labelled 0 in airport sub-network; hence, the ZHDoSE value of $\mathrm{SE} 2$ is 1 , and $C_{0}^{2}=1$. Likewise, SE1 has two subnodes labelled 0 in the airport sub-network and the railway sub-network, so $C_{0}^{1}=2$.

In HSN, the number of shared SEs (NSSE) is the total number of SEs that the sub-nodes in the subway sub-network shared with other SEs. Equation 2 shows the calculation:

$S D_{e}=\sum_{i=1}^{I} N_{S E}^{x_{i}}$

where $S D_{e}$ represents the NSSE of $e$-th SE, and $I$ is the number of sub-nodes in the $e$-th SE on the subway sub-network; $N_{S E}^{x_{i}}$ is the number of SEs that its sub-node $x_{i}$ belongs to the subway sub-network; $x_{i}$ means the $i$-th sub-node in the subway sub-network. For example, SE2 in Figure 1 has sub-nodes labelled $2,3,4$, and 5 in the subway sub-network, sub-node labelled 2 has shared 3 SEs, sub-node labelled 3 has shared 3 SEs, sub-node labelled 4 has shared 4 SEs, and sub-node labelled 5 has shared 3 SEs; hence, the NSSE of SE2 is $13, S D_{2}=13$.

The index of NSSE can well represent the selectivity of SE in a subway station. For example, the starting and ending stations in the subway lines have fewer options than the transfer stations. More precisely, this index can be a representation of subway lines that the SE catches to some extent. Therefore, it may have the potential to affect the topological connectivity of hubs.

The correlation analysis has been conducted to make sure whether ZHDoSE and NSSE are relevant or not. The number of stations $N$ is 287 , and Pearson correlation is 0.091 , which is lower than 0.1. Therefore, the indices of ZHDoSE and NSSE are independent. The index of ZHDoSE could well 
represent the contribution of transport modes, meanwhile, the index of NSSE plays an important role in the contribution of subways.

\subsection{Expression of connectivity}

In HSN, more labelled 0 sub-nodes mean fewer transport modes in SEs; meanwhile, less NSSE of SE shows less connection to other SEs, which means fewer chances to respond to the sudden changes. As a result, in this paper, the connectivity is described as the combination score of ZHDoSE and NSSE. However, the values of ZHDoSE and NSSE dimension have some impacts on the results. Therefore, to overcome these potential effects, four types of combinations are proposed based on two indices. The first two are ZHDoSE combined with NSSE reciprocal under elements normalization or no-normalization. The last two are ZHDoSE reciprocals combined with NSSE, which also considers whether the elements are normalized or not.

\section{Elements normalization}

There are three common methods for normalization, and all of them have advantages under different circumstances. However, to make the values fit into the 0-1 interval, which can effectively avoid the influence of dimensional value, this paper adopts the max-min normalization, and the normalized formula is shown in Equation 3:

$$
x_{\text {norm }}=\frac{x_{i}-x_{\min }}{x_{\max }-x_{\min }}
$$

where $x_{n o r m}$ is the production of normalization, $x_{\max }$, $x_{\min }$ are the maximum and minimum of the whole elements, respectively; and $x_{i}$ is the $i$-th element.

\section{Connectivity of SEs}

As mentioned before, four kinds of combinations of ZHDoSE and NSSE are presented in this paper. Besides, linear fitting is used in this section, because it is convenient for debugging parameters, quick results, and suitable for fewer parameters. Therefore, the four types of construction in this research are shown as follows:

$$
\begin{aligned}
& R s_{S E 1}^{e}=a C_{\text {norm } 0}^{e}+\frac{b}{S D_{\text {norm_e }}} \\
& R s_{S E 2}^{e}=\frac{a}{C_{n o r m 0}^{e}}+b S D_{\text {norm_e }} \\
& R s_{S E 3}^{e}=a C_{0}^{e}+\frac{b}{S D_{e}} \\
& R s_{S E 4}^{e}=\frac{a}{C_{0}^{e}}+b S D_{e}
\end{aligned}
$$

where $R s_{S E 1}^{e}, R s_{S E 2}^{e}, R s_{S E 3}^{e}, R s_{S E 4}^{e}$ are the connectivity of the $e$-th SE; $a, b$ are parameters and $a+b=1$; $C_{\text {norm } 0}^{e}, C_{0}^{e}$, and $S D_{e}, S D_{n o r m \_}$are non-normalization or normalization values, respectively.

\section{CASE STUDY}

\subsection{Hubs in Beijing}

In this paper, the number of hubs in the case study equals the number of subway stations in Beijing. Therefore, based on the Beijing rail transit lines map, a total of 287 subway stations have been studied in this paper. Figure 2 shows the spatial distribution of SEs in Beijing, which is the spatial distribution of subway station locations. Only the Beijing international airport is adopted in the airport sub-network in this case study. Meanwhile, in the subway sub-network, the T2 subway station and T3 subway station are merged into one SE labelled SE1. In addition, four railway stations are considered in the railway sub-network, namely Beijing south railway station, Beijing west railway station, Beijing north railway station, and Beijing railway station. Nine passenger hubs are contained in the HSN model of the Beijing city. Moreover, the numbering principle of SEs is firstly based on the increasing of the number of transport modes from SE1 to SE287. Then, when two hubs have an equal number of transport modes, the sort is according to the sequence of transport mode of airplane, train, highway passenger hubs, and subway. For example, SE1 to SE3 have three transport modes, SE4 to SE12 have two transport modes, and SE 13 to SE 287 have one transport mode.

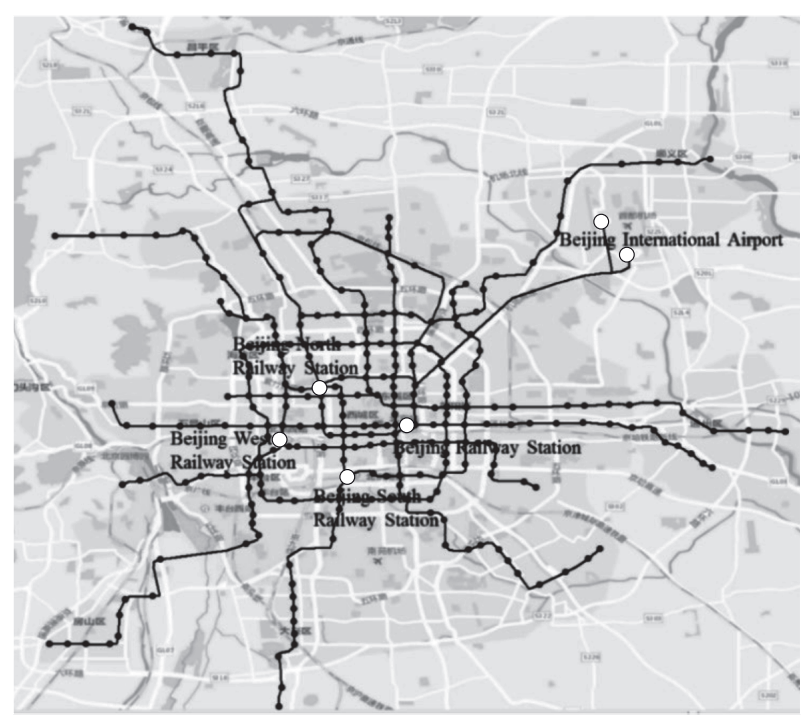

Figure 2 - Spatial distribution map of SEs 


\subsection{Results of topological indices}

The results of ZHDoSE are indicated in Figure 3. From Figure 3 it can be seen that most of the SEs have only one transport mode (subway), i.e. $C_{0}^{e=3}$ and the percentage equals almost $96 \%$. Less than $4 \%$ of urban hubs in this model are stations which support multi-modality, i.e. two or more travel modes. This index represents in a certain sense the number of transport modes.

Figure $3 b$ shows that the majority of SEs that account for 33\% have the NSSE value of 9 in Beijing. These SEs are normal subway stations in the subway sub-network, which are neither transfer stations nor end point stations. Each of them has two normal stations nearby and normal stations have three shared SEs, i.e. 3:3=9. Therefore, three normal stations have nine NSSEs. And then, it is followed by 11 NSSEs which is about $17 \%$. Besides, the maximum

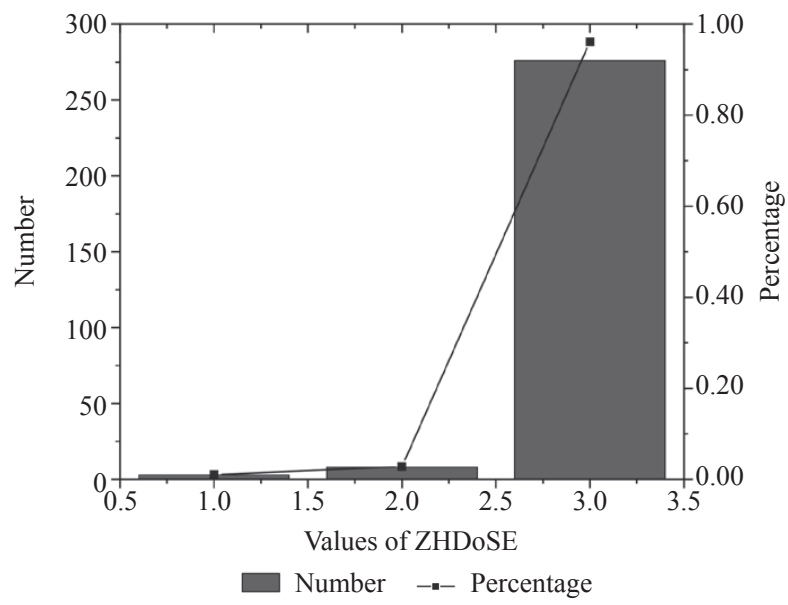

a) ZHDoSE of SES value of NSSE is 23, which is SE5 with the name of Beijing north railway station (Xizhimen station in Line 2, Line 4, and Line 13).

\subsection{Results of connectivity}

Based on the two topological indices and to distinguish the weights of different factors, this paper takes constant $a$ as 0.4 and $b$ as 0.6 according to previous study [36]. The results of four expressions are represented here.

The top ten SEs in Table 1 are selected under the combination of elements normalization and NSSE reciprocal. Depending on the definition of ZHDoSE, it shows clearly that the lower score means higher connectivity. From Table 1 a significant result is that $30 \%$ of SEs (SE1, SE2, and SE3) contain three kinds of transport modes. Furthermore, all SEs support more than two types of modes. Moreover, the top three hubs are all railway stations.

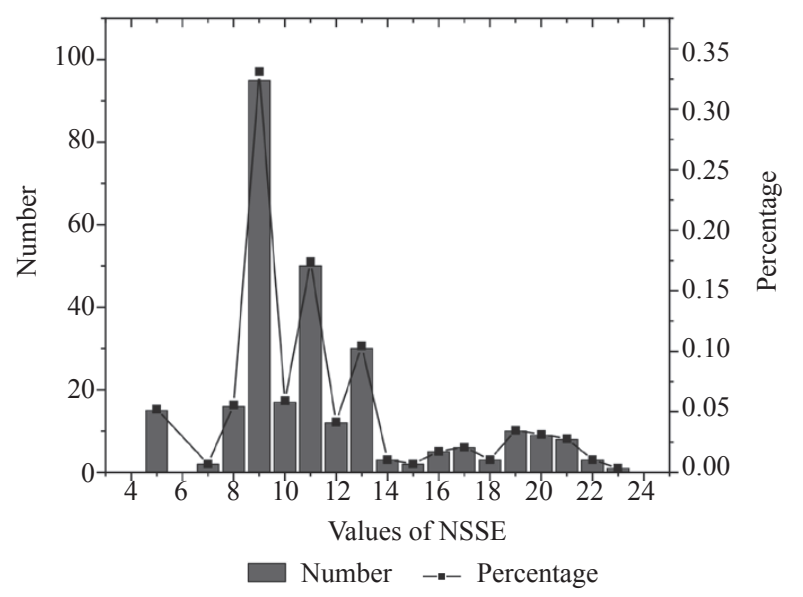

b) NSSE of SES

Figure 3 - Results of ZHDoSE and NSSE

Table 1 - Top ten SEs based on elements normalization and NSSE reciprocal

\begin{tabular}{||c|l|l|c|c|c||}
\hline \hline SE & \multicolumn{1}{|c|}{ Hub name } & \multicolumn{1}{|c|}{ Subway line } & ZHDoSE & NSSE & $R s_{S E 1}^{e}$ \\
\hline \hline SE3 & Beijing west railway station & Lines 7, 9 & 1 & 15 & 0.089 \\
\hline SE2 & Beijing south railway station & Lines 4, 14 & 1 & 13 & 0.128 \\
\hline SE5 & Beijing north railway station & Lines 2, 4, 13 & 2 & 23 & 0.200 \\
\hline SE7 & Bawangfen hub & Lines 1, 14 & 2 & 22 & 0.208 \\
\hline SE6 & Liuliqiao hub & Lines 9, 10 & 2 & 20 & 0.225 \\
\hline SE4 & Beijing railway station & Line 2 & 2 & 13 & 0.328 \\
\hline SE8 & Zhaogongkou hub & Line 5 & 2 & 13 & 0.328 \\
\hline SE10 & Lianhuachi hub & Line 9 & 2 & 12 & 0.352 \\
\hline SE1 & Beijing international airport & Airport Line & 1 & 7 & 0.381 \\
\hline SE11 & Sihui hub & Line 1, Batong & 2 & 11 & 0.382 \\
\hline
\end{tabular}


The result of connectivity under ZHDoSE reciprocal and normalization is shown in Table 2. In this expression, the higher the score of SE, the higher is the connectivity. The prominent characteristic is that each SE of the top ten contains at least two subway lines. Besides, $50 \%$ of SEs have only one transportation mode, but all of SEs have more than two transport modes in Table 1. In addition, as Table 1 shows, the top three hubs are all railway stations.

As mentioned before, non-normalized elements should be taken into consideration. The result of no-normalization and NSSE reciprocal combination are presented in Table 3 which shows the same top ten SEs as Table 1. However, the sorting of the SEs is different; for example, SE1 ranks no. nine in Table 1 but top three in Table 3. In addition, the SEs that have three transport modes are in top three, which is different from Table 1. SE1 in Table 3 ranks in top three but not in Table 1. Moreover, $100 \%$ of top ten SEs have at least two transport modes.

The results of no-normalization and ZHDoSE reciprocal are shown in Table 4. According to the list, all of the top ten SEs have two subway lines, but $60 \%$ of SEs in Table 3 have two subway lines. There are $80 \%$ of them that have one transport mode and $100 \%$ of SEs are transfer stations, but no SE has only one transport mode.

\section{DISCUSSION}

\subsection{Different combinations}

As mentioned above, there are four combinations of connectivity based on two indices. Therefore, discussions on different combinations have been conducted, which can help find out how the transport modes and subway lines perform under different cases.

Firstly, the results of combination of elements normalization and NSSE reciprocal show that three railway stations (Beijing west railway station, Beijing south railway station and Beijing north railway station) rank top three in top ten SEs. The SE4 (Beijing railway station) is not even in the top five, and the difference between SE4 and top three SEs is that the number of its subway lines is lower than of the other railway stations. Moreover, the SE5 (ZHDoSE $=2$ ) has three subway lines ranked behind SE2 and SE3 (ZHEoSE=1), and the value of NSSE is higher than SE2 and SE3. What is more, SE11 seems to contain two subway lines, but it gets low values of NSSE because Line 1 and Line Batong transfer at the same station. However, SE1 (Beijing international airport and passenger hub of the airport) only has one subway line and is endpoint subway station, which has low values of NSSE. In conclusion, compared with the SEs (SE3, SE2, and SE5) and SEs (SE1 and SE11), the ZHDoSE could be more important than the NSSE, which indicates that the number of transport modes makes a much greater contribution than the number of subway lines in HSN.

Secondly, for the ZHDoSE reciprocal under normalization, it can be seen that the top three SEs are still SE3, SE5, and SE2, which fits the results shown in Table 1. However, SE2 and SE5 have changed their rankings, which might be illustrated by the fact that the superiority of ZHDoSE weakens under ZHDoSE reciprocal. Compared to top ten SEs selected by normalization and ZHDoSE reciprocal combination, it might show that ZHDoSE is more critical than NSSE, but it can strengthen the contribution of subway lines because the SEs with more subway lines are selected. However, as the subsequent eight SEs show in this combination, more transport modes have more advantages, which represents higher connectivity. Compared to Table 1, SE1 has three transport modes and the value of NSSE is seven, which is not on the list in Table 2. All SEs shown in Table 2 have more than two subway lines, which indicates that ZHDoSE reciprocal weakens and might promote the contribution of the number of subway lines. Therefore, the subway lines are strengthened by ZHDoSE reciprocal and normalization. However, the number of transport modes does not contribute well in this case.

Thirdly, as for no-normalization under NSSE reciprocal, Table 3 shows the same top ten SEs as shown in Table 1. Of course, it is somewhat different from the element normalization, SE1 ranks in top three. The No. 3 SE in Table 1 is SE5, which now ranks No. 4. For more specifics, the difference between SE1 and SE5 is that SE1 contains three kinds of transport modes, but only one subway line. However, SE5 gathers three subway lines (Line 2, Line 4, and Line 13) and has one fewer transport mode than SE1. Generally, from these results, it can be concluded that the transport modes play a more significant role than the number of subway lines under no-normalization and NSSE reciprocal combination. In addition, no-normalization makes transport modes contribute a lot to the connectivity than the 
Yuan G, et al. Connectivity Contribution to Urban Hub Network Based on Super Network Theory - Case Study of Beijing

Table 2 - Top ten SEs based on elements normalization and ZHDoSE reciprocal

\begin{tabular}{||c|l|l|c|c|c||}
\hline SE & \multicolumn{1}{|c|}{ Name } & \multicolumn{1}{|c|}{ Subway line } & ZHDoSE & NSSE & $R s_{S E 2}^{e}$ \\
\hline \hline SE3 & Beijing west railway station & Lines 7, 9 & 1 & 15 & 0.733 \\
\hline SE5 & Beijing north railway station & Lines 2, 4, 13 & 2 & 23 & 0.700 \\
\hline SE2 & Beijing south railway station & Lines 4, 14 & 1 & 13 & 0.667 \\
\hline SE7 & Bawangfen hub & Lines 1, 14 & 2 & 22 & 0.667 \\
\hline SE6 & Liuliqiao hub & Lines 9, 10 & 2 & 20 & 0.600 \\
\hline SE31 & Guomao Station & Lines 1, 10 & 3 & 22 & 0.567 \\
\hline SE33 & Chegongzhuang Station & Lines 2, 4 & 3 & 22 & 0.567 \\
\hline SE24 & Xidan Station & Lines 1, 4 & 3 & 21 & 0.533 \\
\hline SE28 & Dongdan Station & Lines 1, 5 & 3 & 21 & 0.533 \\
\hline SE29 & Jianguomen Station & Lines 1, 2 & 3 & 21 & 0.533 \\
\hline \hline
\end{tabular}

Table 3 - Top ten SEs based on no-normalization and NSSE reciprocal

\begin{tabular}{||c|l|l|c|c|c||}
\hline SE & \multicolumn{1}{|c|}{ Name } & \multicolumn{1}{|c|}{ Subway line } & ZHDoSE & NSSE & $R s_{\text {SE3 }}^{e}$ \\
\hline \hline SE3 & Beijing west railway station & Lines 7, 9 & 1 & 15 & 0.440 \\
\hline SE2 & Beijing south railway station & Lines 4, 14 & 1 & 13 & 0.446 \\
\hline SE1 & Beijing international airport & Airport Line & 1 & 7 & 0.486 \\
\hline SE5 & Beijing north railway station & Lines 2, 4, 13 & 2 & 23 & 0.826 \\
\hline SE7 & Bawangfen hub & Lines 1, 14 & 2 & 22 & 0.827 \\
\hline SE6 & Liuli Qiao hub & Lines 9, 10 & 2 & 20 & 0.830 \\
\hline SE4 & Beijing railway station & Line 2 & 2 & 13 & 0.846 \\
\hline SE8 & Zhaogongkou hub & Line 5 & 2 & 13 & 0.846 \\
\hline SE10 & Lianhuachi hub & Line 9 & 2 & 12 & 0.850 \\
\hline SE11 & Sihui hub & Line 1, Batong & 2 & 11 & 0.855 \\
\hline
\end{tabular}

Table 4 - Top ten SEs based on no-normalization and ZHDoSE reciprocal

\begin{tabular}{|c|c|c|c|c|c|}
\hline SE & Name & Subway line & ZHDoSE & NSSE & $R s_{S E 4}^{e}$ \\
\hline SE5 & Beijing north railway station & Lines $2,4,13$ & 2 & 23 & 14.000 \\
\hline SE7 & Bawangfen hub & Lines 1,14 & 2 & 22 & 13.400 \\
\hline SE31 & Guomao station & Lines 1,10 & 3 & 22 & 13.333 \\
\hline SE33 & Chegongzhuang station & Lines 2, 4 & 3 & 22 & 13.333 \\
\hline SE24 & Xidan station & Lines 1,4 & 3 & 21 & 12.733 \\
\hline SE28 & Dongdan station & Lines 1,5 & 3 & 21 & 12.733 \\
\hline SE29 & Jianguomen station & Lines 1,2 & 3 & 21 & 12.733 \\
\hline SE36 & Xuanwumen station & Lines 2, 4 & 3 & 21 & 12.733 \\
\hline SE39 & Chongwenmen station & Lines 2,5 & 3 & 21 & 12.733 \\
\hline SE40 & Chaoyangmen station & Lines 2,6 & 3 & 21 & 12.733 \\
\hline
\end{tabular}


normalization under NSSE reciprocal; perhaps the normalization weakens the role of transport modes on the connectivity.

Lastly, compared to the results presented in Table 2 and Table 3, the results in Table 4 of the combination of ZHDoSE reciprocal and no-normalization, emphasises the contribution on the number of subway lines. The contribution of NSSE might be greatly strengthened, because the values of NSSE are descending in Table 4 while they are not in Table 2. Though the SEs of top ten in Table 4 have more than two subway lines, there are no SEs that have three transport modes. Moreover, only two SEs (SE5, SE7) have two kinds of transport modes, and no SE has three kinds of transport mode shown on the list. In addition, compared to Table 3 and Table 4, the value of ZHDoSE has an increasing trend in either table, which shows that the ZHDoSE strengthens under no-normalization. However, the extent of strengthening is affected by the reciprocal, because the SEs whose values of ZHDoSE equal 1 are not shown in Table 4. Moreover, as NSSE descends in Table 4, the ZHDoSE reciprocal under no-normalization might strengthen the NSSE.

Therefore, compared to the results of all four combinations and taking the real situation of urban hubs in the Beijing city into consideration, the results of the paper can be further explained as follows. As for reciprocal cases, the NSSE reciprocal shows more practical identifications on performing subway lines and transport modes than ZHDoSE reciprocal whether under normalization or not. As for normalization cases, the NSSE reciprocal can obtain better results than ZHDoSE reciprocal. Besides, the transport modes have great contribution on connectivity under no-normalization. As this paper shows, the number of transport modes seems more important for connectivity than the number of subway lines.

To conclude, compared to NSSE reciprocal and ZHDoSE reciprocal combination, the ZHDoSE reciprocal might greatly weaken the effect of transport modes on the connectivity and strengthen the subway lines under normalization. In addition, no-normalization might strengthen the contribu- tion of subway lines under ZHDoSE reciprocal. No-normalization of elements always strengthens the contribution of transport modes whether under ZHDoSE or NSSE reciprocal (see Table 5).

In short, the studies in the Beijing city show that the combination of elements normalization and NSSE reciprocal has more practical significance among the rest of three combinations, because it can well reflect the contribution of transport mode and subway lines. As Beijing international airport (SE1) has three transport modes, but only one subway line and a few stations, it ranks the ninth in Table 1.

\subsection{Analysis of parameter $a$}

Based on the previous research, $a$ and $b$ have adopted 0.4 and 0.6 for four combinations in this paper, respectively. However, different values might have different effects. Therefore, different values of parameter $a(b=1-a)$ are discussed in this section. The purpose is to explore the impacts of different parameters on the results. Figure 4 shows numerical simulation results. Axis $Y$ represents the calculation results of the combination of ZHDoSE and NSSE .

It can be seen from the figures that the trend of different lines in Figure $4 a$ seems similar, especially after SE25. With the value of a increasing, the scores of different SEs seem to be rising, but the highest values do not exceed 1. Figure $4 b$ shows more details about SE1 to SE25. However, SE1, SE2, and SE3 have a decreasing tendency with an increase of constant $a$. It is known that three transport modes have these three SEs, which might be explained by the elements normalization that makes the value of ZHDoSE 0. Of course, the tendency changes after SE11 because of the jumping crossover phenomenon in Figure $4 b$. The SEs after SE11 have only one transport mode, which might explain the change. Moreover, in the combination of normalization, some SEs (SE12, SE47, SE67, and so on) contain endpoint stations that have the same score equalling 1, though parameter a varies from 0.01 to 0.99 . Furthermore, as parameter $a$ is higher than 0.95 , the scores of SEs after No. 11 that has only one transport mode (subway) are close to 1 . Therefore, under the combination of NSSE reciprocal with elements

Table 5 - Strengthening results of different combinations

\begin{tabular}{||l|l|l||}
\hline & \multicolumn{1}{|c||}{ ZHDoSE reciprocal } & \multicolumn{1}{|c|}{ NSSE reciprocal } \\
\hline \hline Normalization & Subway lines & -- \\
\hline No-normalization & Subway lines; Transport modes & Transport modes \\
\hline
\end{tabular}


normalization, when parameter $a$ is large (over 0.95), the connectivity of most SEs in the network tends to be more homogeneous, but it does not work for other SEs which have more than two transport modes.

Figure 5 shows the simulation results of NSSE reciprocal without elements of normalization. Figure $5 a$ shows the trends: as parameter $a$ increases the score seems to be rising, especially the SEs after SE25. The scores are over 1 that is different from the normalization case, which might be caused by normalization methods. Compared to the results shown in Figure 4, the results verify that elements normalization weakens the contribution of transport modes, because SE1, SE2, and SE3 do not drop with parameter $a$ increasing. In addition, there is an increasing trend when $a$ equals 0.95 and 0.99 . Moreover, there are obvious three steps (first step:

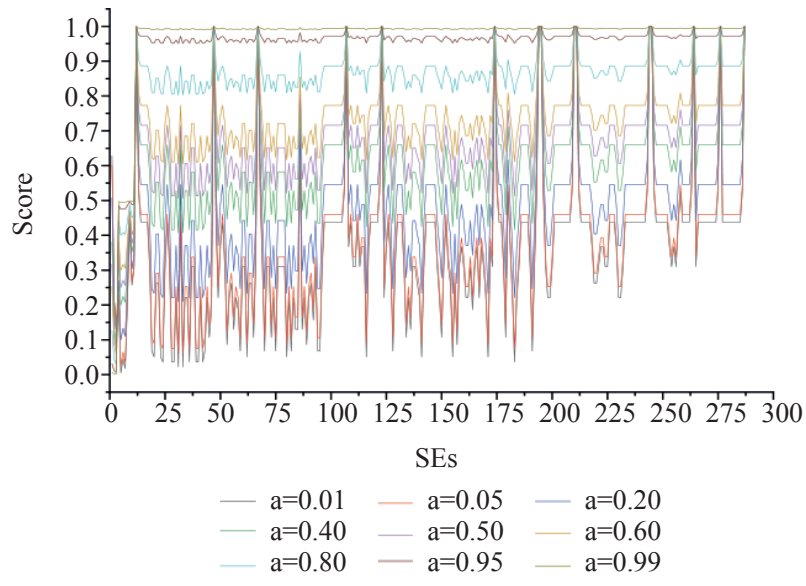

a) SEs score polygonal map
SE1 to SE3, second step: SE4 to SE12, and third step: SE13 to SE287) in Figure 5b, which is also actually the boundary of the number of transport modes. The higher the parameter $a$, the more obvious are the three-stage phenomena. As the lower score means more trip ways it contains, for instance, SEs have three kinds of transport modes in the first step, two transport modes in the second step, and only one in the third step. In addition, the value of constant $a$ is no more than 0.05 , which tends to achieve the homogeneity of SEs in HSN, because the score of all SEs features no big fluctuation. In conclusion, under no-normalization, when parameter $a$ is lower than 0.05 , it has the tendency to make the score of all SEs converge.

In conclusion, parameter $a$ has great influence on the results in the normalization case, but it has fewer impacts on no-normalization case. In addition,

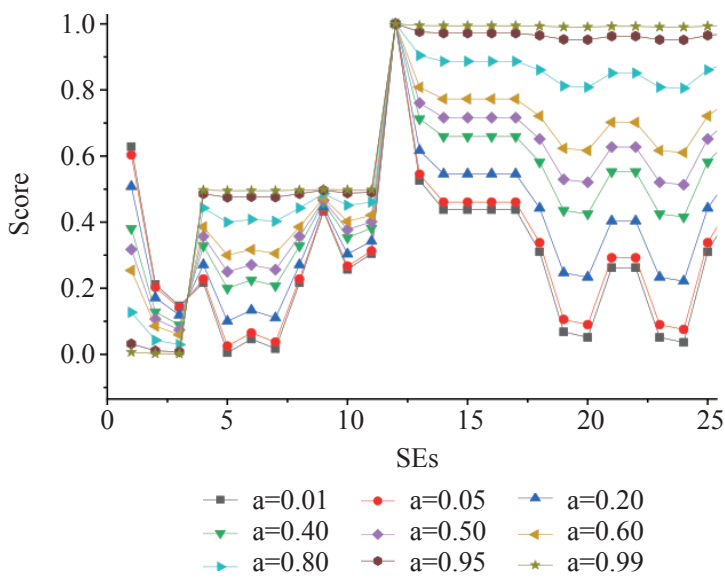

b) SEs score locally enlarged point map

Figure 4 - Results of parameter a under NSSE reciprocal with elements normalization

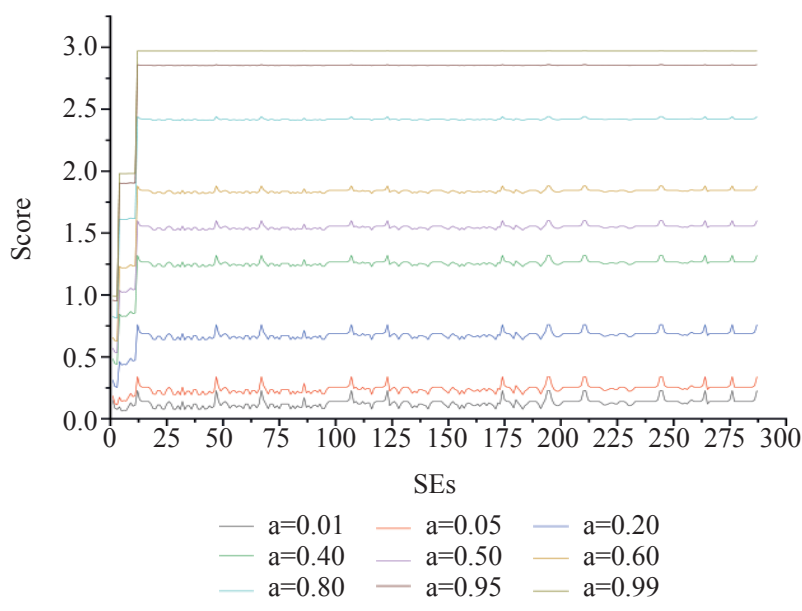

a) SEs score polygonal map

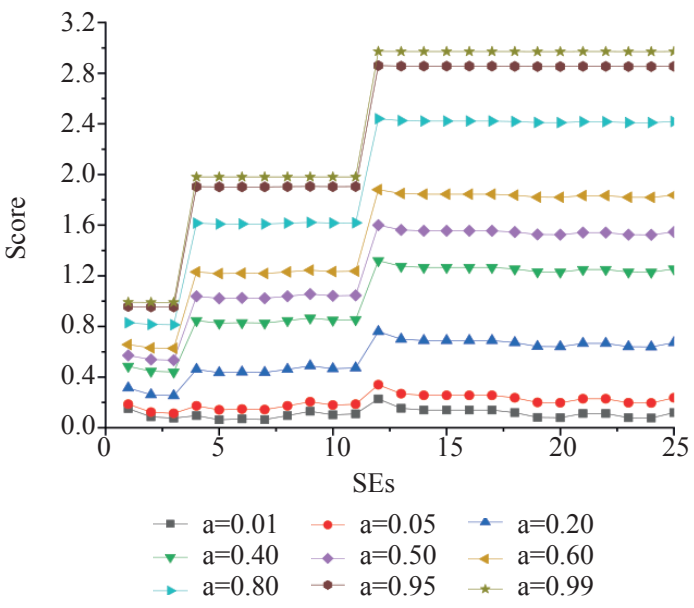

b) SEs score locally enlarged point map

Figure 5 - Results of parameter a under NSSE reciprocal without elements normalization 
when value $a$ is lower than 0.05 , the sorting of all SEs will somewhat change under no-normalization. Moreover, two situations can get more differentiated ranking of SEs in HSN. One is that parameter $a$ is lower than 0.95 while elements have been normalized, the other is that parameter $a$ is higher than 0.05 in case of no-normalization.

\section{CONCLUSION}

In this paper a research has been conducted on the urban transport hubs that have multimodal travel means, and the HSN framework is proposed based on SNT. Further, two indices are presented and four combinations are discussed in this paper. The two indices are proposed to represent the number of transport modes and subway lines. In addition, the results of four expressions are discussed and have been validated in the Beijing case. The main findings are summarized as follows:

1) HSN is built based on the theory of super network. Two structural features (ZHDoSE and NSSE) are proposed to study the connectivity of the hubs. Besides, a case study of Beijing is conducted to analyse the topological characteristics of the hubs in Beijing. It has been proven that SNT can be well applied in the field of urban hubs.

2) In HSN four expressions of connectivity are proposed based on the indices of ZHDoSE and NSSE. The finding is that the transport modes contribute a lot to the connectivity of hubs. Besides, no-normalization strengthens the contribution of the transport mode and subway lines in ZHDoSE reciprocal case. Moreover, no-normalization strengthens the transport modes both in the case of ZHDoSE and NSSE reciprocal. Thus, the combination of no-normalization and NSSE reciprocal performs better than other combinations.

3) The effect of parameter $a$ on the results has been preliminarily studied. Parameter $a$ has more influence on normalization than no-normalization in NSSE reciprocal. However, it has fewer impacts on no-normalization when parameter $a$ is higher than 0.05 . Therefore, when parameter $a$ is higher than 0.05 and combined with no-normalization or when it is lower than 0.95 and combined with normalization, the rank of SEs in HSN can be well recognized.
This task makes some progress on the application of SNT in the transportation field and the research of connectivity of urban transport hubs based on SNT. However, due to the limitation of work, a lot of details have not been analysed. For example, the results of four combinations need to be analysed in detail, and not just the top ten of all SEs, and the experiments on various parameters. Besides, more indices should be explored based on HSN and more characteristics should be studied in the future work.

\section{ACKNOWLEDGEMENTS}

The authors would like to acknowledge the financial support for this study provided by the Science and Technology Program of Beijing (Z191100002519002), Beijing Municipal Education Commission Science and Technology Program General Project (KM202110005002), the China Postdoctoral Science Foundation (Grant No. 2018M641138), the Beijing Postdoctoral Research Foundation (ZZ2019-108), the Basic Research Foundation Project of Beijing University of Technology (038000546319519), and the Chaoyang District Postdoctoral Research Foundation (Q1038001201901).

袁广, 在读博士 $1,3,4$

电子邮箱: yguang@emails.bjut.edu.cn

孔德文, 博士 $1,3,4$

电子邮箱: kongdw@bjut.edu.cn

孙立山, 博士 $1,3,4$

(通讯作者)

电子邮箱: 1ssun@bjut.edu.cn

罗薇, 博士 ${ }^{2}$

电子邮箱: luowei@bucea.edu.cn

许琰, 博士 $1,3,4$

电子邮箱: yxu@bjut.edu.cn

1 北京工业大学北京市交通工程重点实验室

北京市朝阳区平乐园100号

2 北京建筑大学

北京市大兴区永源路 15 号

3 城市轨道交通北京实验室, 北京市朝阳区平乐园100号

4 数字社区教育部工程研究中心,

北京市朝阳区平乐园100号

\section{基于超级网络理论的城市枢纽网络连通性贡}

献: 以北京市为例

摘要

随着我国城市化的快速发展, 出行方式和城市客 运枢纽数量不断增加, 逐步形成城市多层次、多属 性的交通枢纽网络。同时, 超级网络理论 ( SNT) 在展示多层交通枢纽方面具有优势。本文旨在为研 究枢纽潜在的连通性贡献提供一个新的视角。基于 枢纽超级网络 (HSN) 的拓扑特征对城市交通枢纽 
进行排序。基于超边 $(S E)$ 本文提出了的两个指 标: 零中心性 $(Z H D o S E)$ 和共享超边数 (NSSE) 。然后, 以北京市为例, 研究了交通方式和地铁线 路对连通性的影响。结果表明, 元素非归一化增强 了交通方式和地铁线路对连通性的贡献。此外，传 输方式对连通性有很大的贡献。然而, 元素归一化 在ZHDoSE倒数下增强了地铁线路数量贡献。另外, 在 $H S N$ 中, ZHDoSE和NSSE的不同权重对SEes的识 别结果有不同的影响。

\section{关键词: 超网络; 超边; 交通枢纽; 连通性}

\section{REFERENCES}

[1] Shen RG, Pei YL. A Parking Demand Forecasting Method for Urban Comprehensive Passenger Transport Hub Oriented High-Speed Rail. Adv Mater Res. 2012;524-527: 828-31. DOI: 10.4028/www.scientific. net/AMR.524-527.828

[2] Paredes R, Dueñas-Osorio L, Meel KS, Vardi MY. Principled Network Reliability Approximation: A Counting-Based Approach. Reliab Eng Syst Saf. 2019;191: 106472. DOI: 10.1016/j.ress.2019.04.025

[3] Li Z, Jin C, Hu P, Wang C. Resilience-based transportation network recovery strategy during emergency recovery phase under uncertainty. Reliab Eng Syst Saf. 2019;188: 503-514. DOI: 10.1016/j.ress.2019.03.052

[4] Leobons CM, Gouvêa Campos VB, De Mello Bandeira RA. Assessing Urban Transportation Systems Resilience: A Proposal of Indicators. Transp Res Procedia. 2019;37: 322-329. DOI: 10.1016/j.trpro.2018.12.199

[5] Sun L, Huang Y, Chen Y, Yao L. Vulnerability assessment of urban rail transit based on multi-static weighted method in Beijing, China. Transp Res Part A Policy Pract. 2018;108: 12-24. DOI: 10.1016/j.tra.2017.12.008

[6] Takebayashi M. Managing airport charges under the multiple hub network with high speed rail: Considering capacity and gateway function. Transp Res Part A Policy Pract. 2018;112: 108-123. DOI: 10.1016/ j.tra.2018.01.011

[7] Xiao X-M, Jia L-M, Wang Y-H. Correlation between heterogeneity and vulnerability of subway networks based on passenger flow. J Rail Transp Plan Manag. 2018;8: 145-157. DOI: 10.1016/j.jrtpm.2018.03.004

[8] Sun D, Guan S. Measuring vulnerability of urban metro network from line operation perspective. Transp Res Part A Policy Pract. 2016;94: 348-359.

[9] Strano E, Shai S, Dobson S, Barthelemy M. Multiplex networks in metropolitan areas: Generic features and local effects. $J$ R Soc Interface. 2015;12(111): 20150651. DOI: 10.1098/rsif.2015.0651

[10] Chen D, Yang Z. Systematic optimization of port clusters along the Maritime Silk Road in the context of industry transfer and production capacity constraints. Transp Res Part E Logist Transp Rev. 2018;109: 174-189. DOI: 10.1016/j.tre.2017.11.007

[11] Acer UG, Giaccone P, Hay D, Neglia G, Tarapiah S. Timely data delivery in a realistic bus network. IEEE Trans Veh Technol. 2012;61(3): 1251-1265. DOI: 10.1109/TVT.2011.2179072

[12] Rodríguez-Núñez E, García-Palomares JC. Measuring the vulnerability of public transport networks. $J$ Transp Geogr. 2014;35: 50-63. DOI: 10.1016/j.jtrangeo.2014.01.008
[13] Mattsson LG, Jenelius E. Vulnerability and resilience of transport systems - A discussion of recent research. Transp Res Part A Policy Pract. 2015;81: 16-34. DOI: 10.1016/j.tra.2015.06.002

[14] Reggiani A, Nijkamp P, Lanzi D. Transport resilience and vulnerability: The role of connectivity. Transp Res Part A Policy Pract. 2015;81: 4-15. DOI: 10.1016/ j.tra.2014.12.012

[15] Ortega A, Stegmann T, Benet L. Robustness of optimal transport in disordered interacting many-body networks. Phys Rev E. 2018;arXiv:1803.05974. DOI: 10.1103/ PhysRevE.98.012141

[16] Corman F, Quaglietta E, Goverde RMP. Automated real-time railway traffic control: An experimental analysis of reliability, resilience and robustness. Transp Plan Technol. 2018;41(4): 421-447. DOI: 10.1080/03081060.2018.1453916

[17] Boonzajer Flaes DE, Stopka J, Turtaev S, De Boer JF, Tyc T, Čižmár T. Robustness of Light-Transport Processes to Bending Deformations in Graded-Index Multimode Waveguides. Phys Rev Lett. 2018;120(23). DOI: 10.1103s/PhysRevLett.120.233901

[18] M'Cleod L, Vecsler R, Shi Y, Levitskaya E, Kulkarni S, Malinchik S, Sobolevsky S. Vulnerability of Transportation Networks: The New York City Subway System under Simultaneous Disruptive Events. Procedia Comput Sci. 2017;119: 42-50. DOI: 10.1016/j.procs.2017.11.158

[19] Sheffi Y. Urban transportation networks: Equilibrium analysis with mathematical programming methods. Prentice-Hall; 1984.

[20] Nagurney A, Wakolbinger T. Supernetworks: An Introduction to the Concept and its Applications with a Specific Focus on Knowledge Supernetworks. Int J Knowledge, Cult Chang Manag Annu Rev. 2005;4(1). DOI: 10.18848/1447-9524/cgp/v04/50227

[21] Ma N, Liu Y. SuperedgeRank algorithm and its application in identifying opinion leader of online public opinion supernetwork. Expert Syst Appl. 2014;41: 1357 1368. DOI: 10.1016/j.eswa.2013.08.033

[22] Feng HU. An evolving hypernetwork model and its properties. Sci Sin. 2013;43(1): 16-22. DOI: 10.1360/13201287

[23] Wang G, Liu Y, Li J, Tang X, Wang H. Superedge coupling algorithm and its application in coupling mechanism analysis of online public opinion supernetwork. Expert Syst Appl. 2015;42: 2808-2823. DOI: 10.1016/ j.eswa.2014.11.026

[24] Zhao L, Zhang H, Wu W. Knowledge service decision making in business incubators based on the supernetwork model. Phys A Stat Mech Its Appl. 2017;479: 249-264. DOI: 10.1016/j.physa.2017.03.013

[25] Yamada T, Febri Z. Freight transport network design using particle swarm optimisation in supply chain-transport supernetwork equilibrium. Transp Res Part E Logist Transp Rev. 2015;75: 164-187. DOI: 10.1016/ j.tre.2015.01.001

[26] Feng Z, Wang Z, Chen Y. The equilibrium of closed-loop supply chain supernetwork with time-dependent parameters. Transp Res Part E Logist Transp Rev. 2014;64: 1-11. DOI: 10.1016/j.tre.2014.01.009.

[27] Zhang X. Multilayer Networks Science: Concepts, 
Theories and Data. Complex Syst Complex Sci. 2015;12: 103-107.

[28] Fang J. From a single network to "network of networks" development process: Some discussion on the exploration of the maltilayer supernetwork model and challenges. Complex Systems and Complexity Science. 2016;13(1): 40-47.

[29] Liu Q, Fang J, Li Y. Research on hierarchical hyper network model based on unified hybrid network theory framework. Complex Systems and Complexity Science. 2016;13(1): 84-90.

[30] Ma J, Wang S, Jian R, Sun L. Using Point of Interest Data from Electronic Map to Predict Transit Station Rideship. Transportation Research Board 93 ${ }^{\text {rd }}$ Annual Meeting, 12-16 Jan. 2014, Washington DC, USA; 2014.

[31] Beijing Transport Institute. 2019 Beijing Transportation Annual Report. Beijing; 2019.

[32] Liu Y, Li Q, Tang X, Ma N, Tian R. Superedge prediction: What opinions will be mined based on an opinion supernetwork model?. Decis Support Syst. 2014;64: 118-129. DOI: 10.1016/j.dss.2014.05.011

[33] Mamun SA, Lownes NE. Access and connectivity tradeoffs in transit stop location. Transp Res Rec. 2014;2466: 1-11. DOI: $10.3141 / 2466-01$

[34] Wei P, Chen L, Sun D. Algebraic connectivity maximization of an air transportation network: The flight routes' addition/deletion problem. Transp Res Part E Logist Transp Rev. 2014;61: 13-27. DOI: 10.1016/ j.tre.2013.10.008

[35] Spiers G, Wei P, Sun D. Algebraic connectivity optimization of the air transportation network. 2012 American Control Conference (ACC), 27-29 June 2012, Montreal, QC, Canada; 2012. p. 1702-1707. DOI: 10.1109/ acc.2012.6314649

[36] Gao YL, Chen SM, Nie S, Ma F, Guan JJ, Robustness analysis of interdependent networks under multiple-attacking strategies. Phys A Stat Mech Its Appl. 2018;496: 495-504. DOI: 10.1016/j.physa.2017.12.085 